\title{
AGENESIA RENAL DERECHA Y ABOCAMIENTO ECTÓPICO DEL URÉTER EN DILATACIÓN QUÍSTICA DE LA VESÍCULA SEMINAL
}

\author{
M. PASCUAL SAMANIEGO, J. EGEA CAMACHO, J.R. CORTIÑAS GONZÁLEZ, \\ J. CALLEJA ESCUDERO, M.D. RIVERO MARTÍNEZ, E. FERNÁNDEZ DEL BUSTO
}

Servicio de Urología. Hospital Clínico Universitario. Valladolid.

Actas Urol Esp. 28 (9): 688-693, 2004

\section{RESUMEN}

\section{AGENESIA RENAL DERECHA Y ABOCAMIENTO ECTÓPICO DEL URÉTER EN DILATACIÓN QUÍSTICA} DE LA VESÍCULA SEMINAL

La asociación de una dilatación quística de la vesícula seminal con agenesia renal ipsilateral, fue descrita por primera vez por Zinner en 1914, constituyendo una anomalía congénita muy poco frecuente. La presencia añadida de la desembocadura ectópica del uréter en dicha vesícula seminal quística resulta aún más excepcional. En estas circunstancias, la clínica suele presentarse a partir de los 15 años de edad, debido a la distensión de la vesícula por las secreciones seminales, siendo el disconfort perineal y las epididimitis de repetición las formas de presentación más frecuentes.

Ante el hallazgo clínico-radiológico de una masa quística pélvica, es obligada la revisión del tracto urinario superior. La resonancia magnética (RM) se muestra como la mejor técnica de imagen para este objetivo, así como para precisar la localización del abocamiento ectópico ureteral en la vesícula seminal.

Aportamos un nuevo caso de esta infrecuente malformación congénita en el que únicamente la RM entre todas las pruebas de imagen, permitió un claro diagnóstico preoperatorio y una adecuada planificación quirúrgica.

PALABRAS CLAVE: Ectopia ureteral. Agenesia renal. Quiste de vesícula seminal. Resonancia magnética.

\section{ABSTRACT \\ RIGHT RENAL AGENESIS AND URETER ECTOPIC ABOUCHEMENT IN CYSTIC DILATION OF SEMINAL VESICLE}

The association of a seminal vesicle cyst with ipsilateral renal agenesis was initially reported in 1914 by Zinner, and it is a very rare congenital anomaly. The association of this anomaly with an ectopic ureter entering the seminal vesicle cyst is even less common. In these cases, clinical symptoms appear in patients 15 years or older due to the distention of the seminal vesicle caused by the secretions of the reproductive tract. Perineal discomfort and recurrent epididymitis are the most often presentations.

Urogenital upper tract image studies are mandatory in the management of a cystic pelvic mass and magnetic resonance imaging (MRI) is the best of this radiographic studies, that also reveals the ectopic ureter draining into the seminal vesicle.

We report an additional case of this rare congenital anomaly where only MRI provided a correct preoperative diagnosis and a right surgical approach.

KEY WORDS: Ectopic ureter. Renal agenesis. Seminal vesicle cyst. Magnetic resonance imaging. 
$\mathrm{L}$ a dilatación quística de la vesícula seminal se presenta en menos del 0,005\% de la población ${ }^{1}$, asociándose una agenesia renal ipsilateral en las dos terceras partes de estos pacientes. Un $28 \%$ de los casos de desembocadura ectópica del uréter ocurren en la vesícula seminal ${ }^{2}$.

Embriológicamente, la yema ureteral surge del extremo caudal del conducto mesonéfrico entre la $4^{\mathrm{a}}$ y $7^{\mathrm{a}}$ semana de gestación ${ }^{3}$. Además del uréter, el mesonefros da origen en su involución a otras dos estructuras; el conducto de Müller del que se desarrollará el utrículo prostático y los apéndices testiculares, y el conducto de Wolff que dará lugar al tracto genital interno en el varón ${ }^{4}$. Inicialmente el uréter y el conducto de Wolff forman un conducto excretor común cuya zona distal será reabsorbida por el seno urogenital para formar el trígono vesical ${ }^{5}$. Pero en la $12^{\mathrm{a}}-13^{\mathrm{a}}$ semana de gestación, se produce un crecimiento diferenciado de la vesícula seminal separándose del uréter en una posición más caudal y medial ${ }^{6}$. El desarrollo precoz del brote ureteral demasiado craneal, ocasiona un retraso en la reabsorción distal, con una desembocadura ectópica en el trígono, el cuello vesical, la uretra prostática, el conducto eyaculador, el deferente o la vesícula seminal $^{5}$. Además, cranealmente, el esbozo ureteral alcanza el blastema renal con predominio de elementos mesonefróticos en vez de tejido metanefrogénico, siendo la consecuencia la disgenesia renal ipsilateral ${ }^{7}$.

A su vez, el desarrollo anormal de la porción distal del conducto mesonéfrico condiciona la atresia congénita del conducto eyaculador, cuya consecuencia es la dilatación quística de la vesícula seminal, que se asocia a la desembocadura ectópica del uréter en dicha vesícula en aproximadamente un 36\% de los casos ${ }^{8}$.

La clinica, si se presenta, es diferente según la localización de la ectopia ureteral. En el tracto urinario, es más frecuente la infección urinaria y el dolor lumbar en un paciente prepuberal, mientras que en el tracto seminal, será la producción seminal post-puberal la que condicione una dilatación de la vesícula, con dolor perineal, eyaculatorio $u$ orquiepididimitis ${ }^{9}$.

Entre todas las pruebas de imagen, la RM permite un diagnóstico definitivo de forma no invasiva.

\section{CASO CLÍNICO}

Varón de 34 años, con antecedente de herniorrafia inguinal e hidrocelectomía derechas, que consultó por dolor continuo de instauración progresiva en la fosa iliaca derecha, no irradiado, que aumentaba con la deambulación. No presentaba clinica miccional ni de la esfera sexual. El dolor se reproducía con la palpación profunda de la fosa iliaca derecha y al realizar el tacto rectal se apreciaba un abombamiento elástico paraprostático derecho no doloroso. El estudio sistemático y bioquímico de sangre fue normal, al igual que el análisis de orina. También se solicitó serología hidatídica con resultado positivo para IgG y negativo para IgE. El único dato relevante del seminograma fue el volumen de $2 \mathrm{ml}$ en el limite inferior de la normalidad.

La ecografía abdominal no identificó el riñón derecho en su correspondiente fosa lumbar, pero si la existencia de una masa quística en contacto con la pared lateral derecha vesical y con la próstata, ambas de características normales, sugiriendo la posibilidad diagnóstica de un riñón ectópico pelviano pielonefrítico crónico. Sin embargo, la localización de dicha imagen por ecografía transrectal, se correspondía con el área de la vesícula seminal derecha, que debido a su disposición anteroposterior, entraba en íntimo contacto con la pared lateral del recto. La rectoscopia fue normal.

Se realizó punción y aspiración transrectal ecodirigida de dicha masa sin encontrarse presencia de espermatozoides en el líquido obtenido, ni presencia de parásitos o serología compatible con hidatidosis. Así mismo se realizó biopsia de su pared, siendo el informe anatomopatológico de tejido fibroso. A pesar de la profilaxis antibiótica y de la preparación intestinal, el paciente presentó una infección yatrogénica por Streptococcus sanguis resistente al tratamiento conservador, que precisó el drenaje del contenido purulento mediante punción ecodirigida por vía abdominal. Una vez superado el episodio, se realizó la inyección de contraste por el catéter manteniendo el radiólogo la posibilidad diagnóstica de un riñón derecho ectópico anulado funcionalmente, debido a la disposición de cavidades dilatadas confluentes que impresionaban como cálices renales (Fig. 1). 


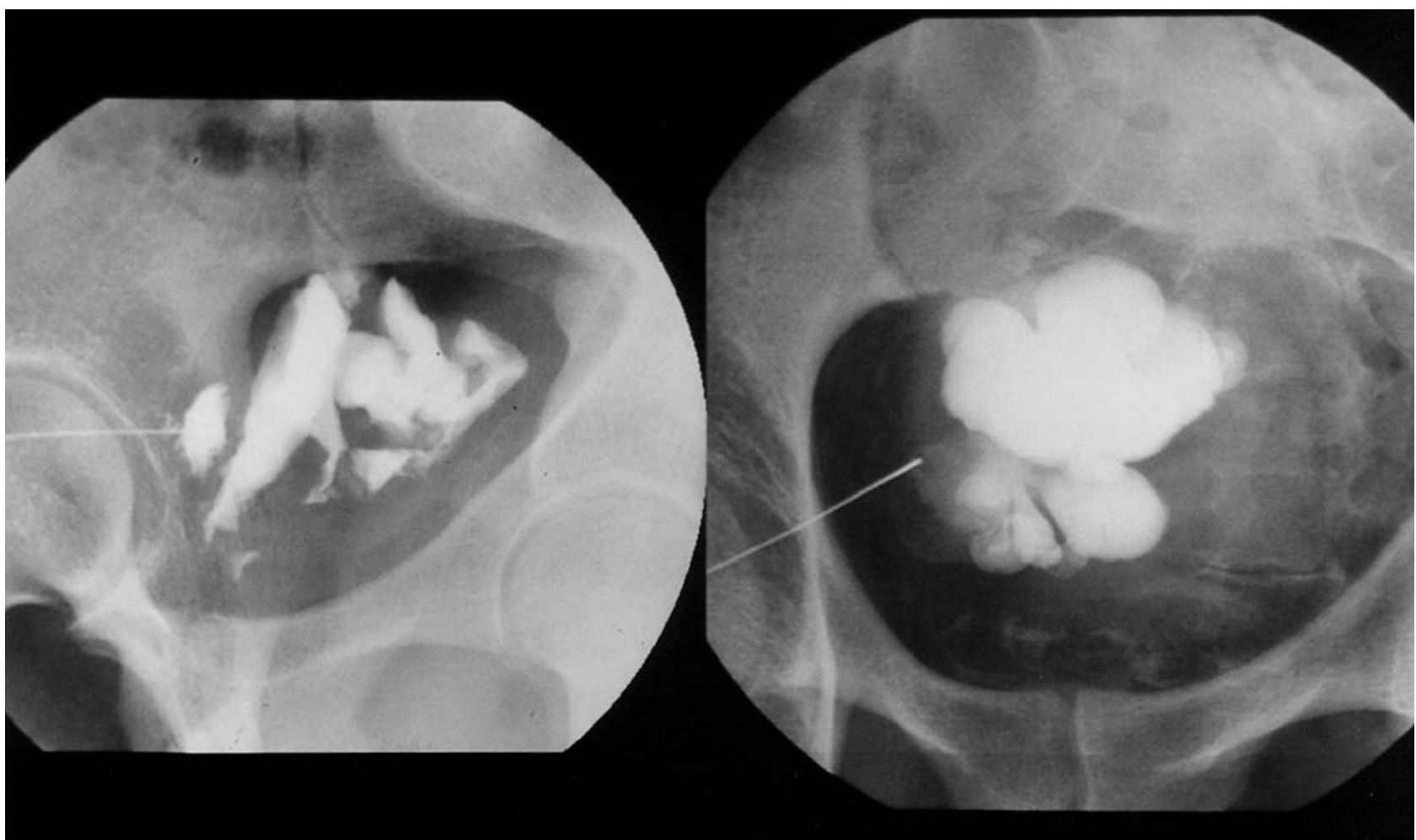

FIGURA 1. Quistografía pélvica. Dilatación de cavidades que confluyen simulando una vía urinaria superior ectópica pelviana.

Por otro lado, la tomografia computerizada (TC) informaba de ausencia renal derecha, relacionando la masa quística paravesical con un posible quiste de la vesícula seminal.

La uretrocistoscopia no identificó el meato ureteral derecho en posición ortotópica ni ectópica, presentando el hemitrígono derecho un abombamiento regular por compresión extrínseca. La gammagrafía renal tampoco detectó tejido renal captante en el área pélvica. Finalmente se recurrió a la resonancia magnética (RM) que fue la única técnica capaz de aclarar el diagnóstico, al detectar en la fosa renal derecha una imagen compatible con un riñón atrófico / displásico de $4 \mathrm{~cm}$ de diámetro, siendo el uréter ectásico en todo su recorrido con inserción distal ectópica en la vesícula seminal derecha. Ésta presentaba una transformación quística, continuándose con un conducto eyaculador dilatado hasta su desembocadura en la uretra prostática (Figs. 2 y 3).

Utilizando un abordaje retroperitoneal por incisión de Gibson, se realizó la extirpación en bloque del tejido disgenésico renal, el uréter y la vesícula seminal con dilatación quística (Fig. 4) con postoperatorio favorable, permaneciendo el paciente asintomático en la actualidad.

El informe anatomopatológico reveló en la localización del riñón, la sustitución del mismo por un tejido conectivo fibroso con granulomas xantohistiocitarios y dilataciones quísticas revestidas por un epitelio columnar simple ciliado y pigmentado. La vesícula seminal presentaba una hiperplasia muscular de su pared.

Diagnóstico definitivo: Agenesia renal derecha con reacción xantogranulomatosa y desembocadura ectópica del uréter en una vesícula seminal quística.

\section{DISCUSIÓN}

La presentación de un uréter ectópico ocurre en $1 / 1900$ personas ${ }^{10}$, siendo 6 veces más frecuente en la mujer. En el varón, la ectopia ureteral no se localiza por debajo del esfinter urinario externo, por lo que no se manifestará clínicamente como incontinencia urinaria ${ }^{11}$. Durante el desarrollo embriológico, si el origen del brote ureteral es demasiado caudal, la disposición lateral del uréter puede condicionar la existencia de reflujo vesicoureteral. Si por el contrario es demasiado craneal, el meato 


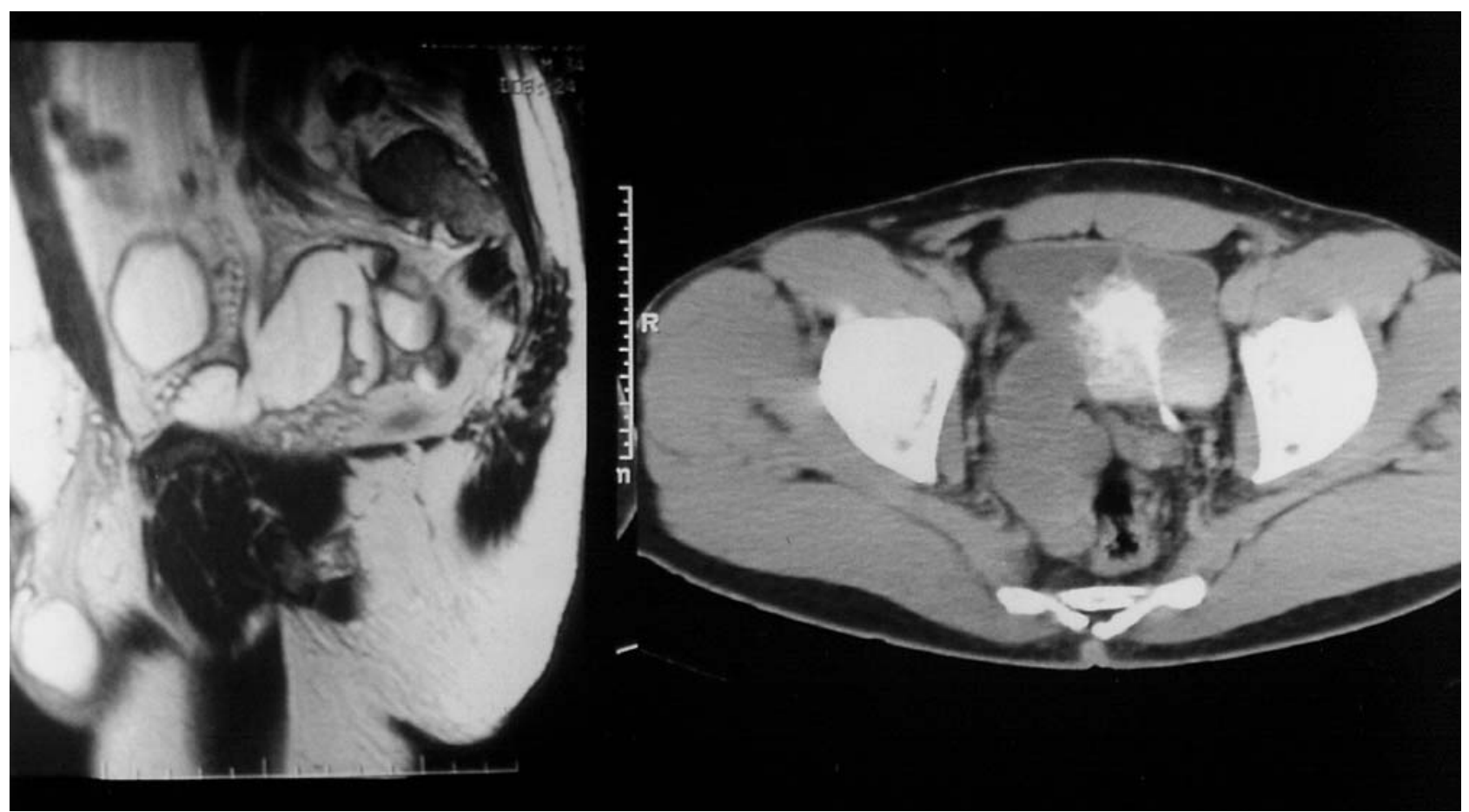

FIGURA 2. RM. Corte sagital que muestra el uréter derecho ectásico desembocando en la vesícula seminal con dilatación quistica. Corte transversal donde se aprecia la disposición anteroposterior de la vesícula seminal quistica para y retrovesical.

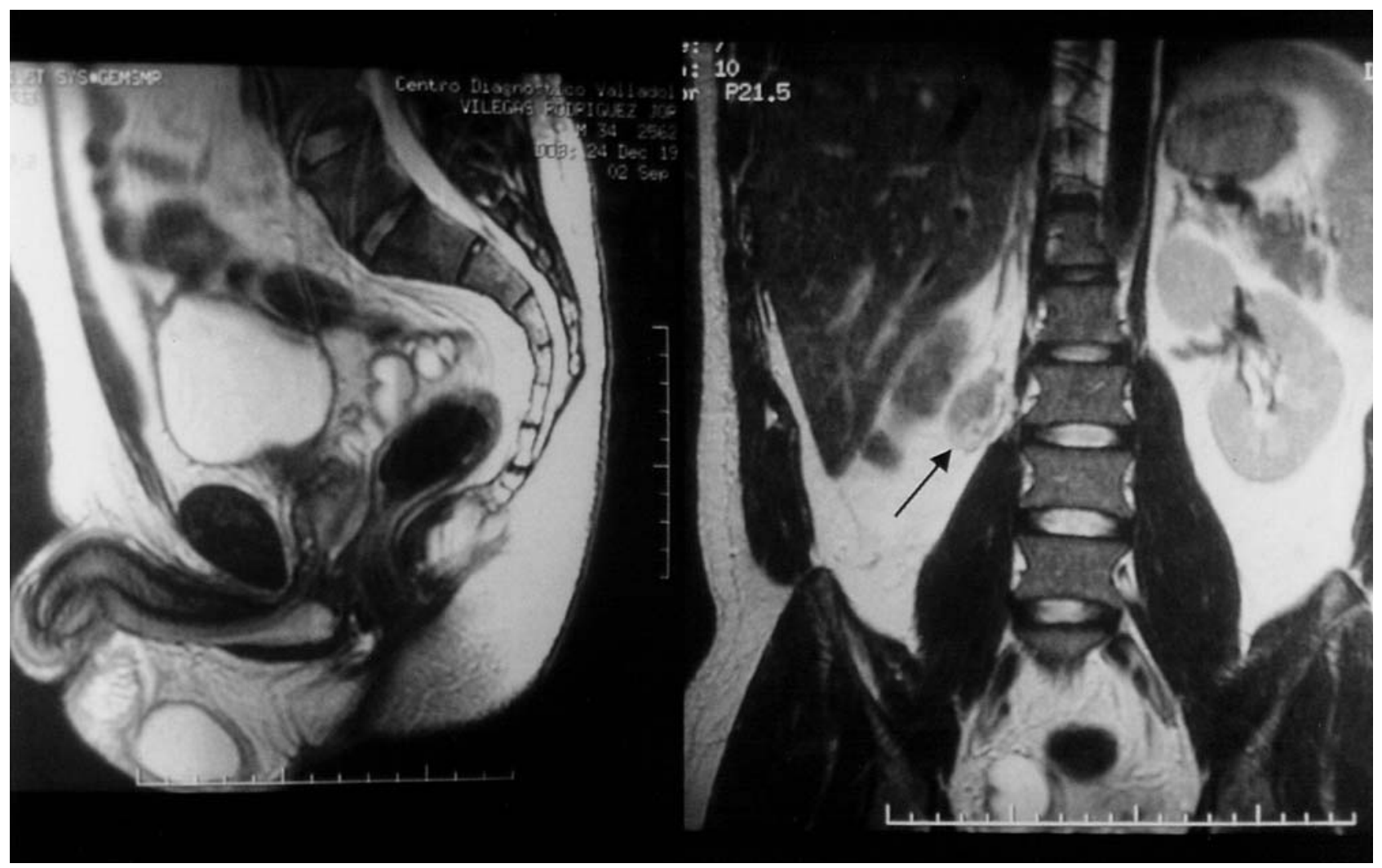

FIGURA 3. RM. Corte sagital: ectasia del conducto eyaculador en continuidad con la vesícula seminal quistica. Corte frontal: la flecha señala el tejido disgenésico que sustituye al riñón derecho. 


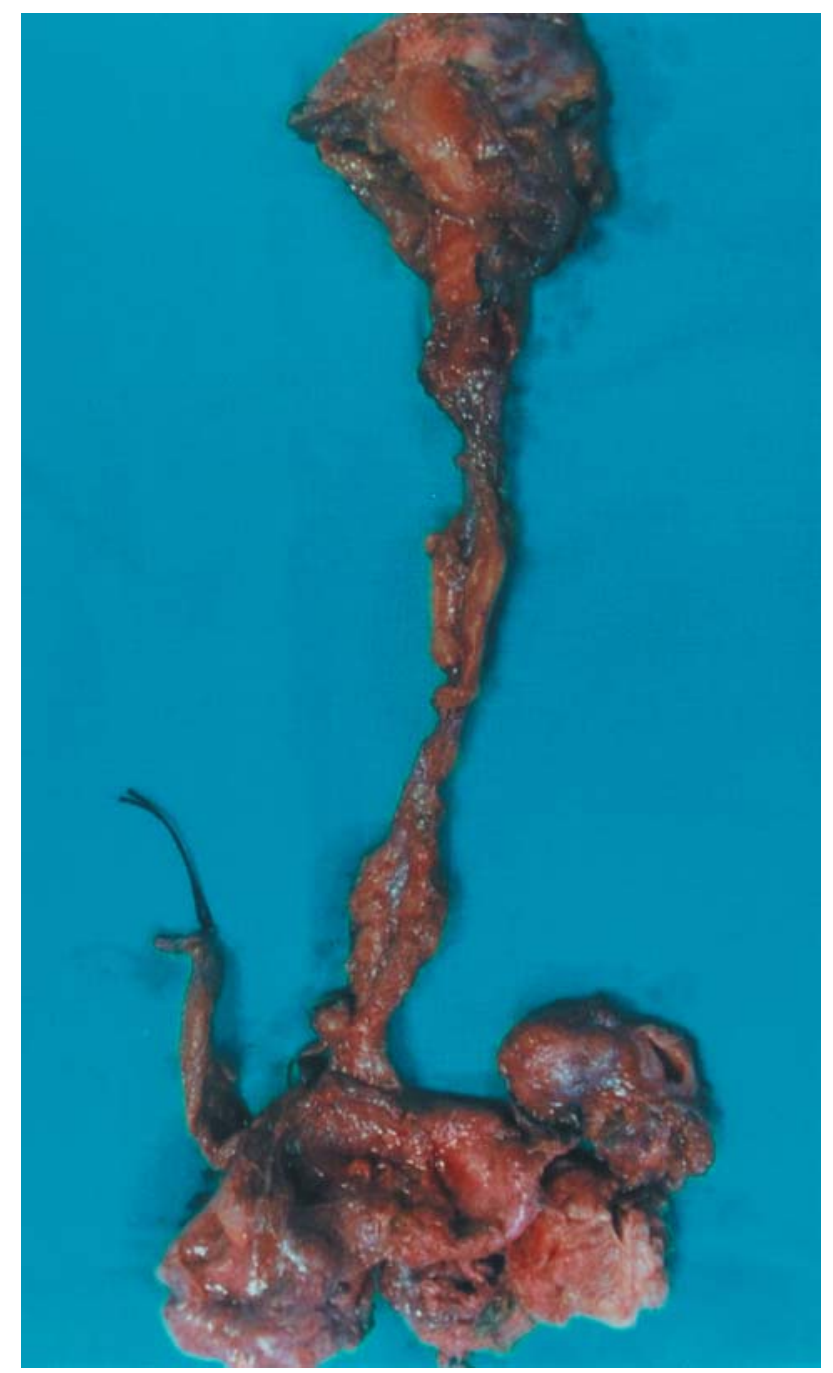

FIGURA 4. Pieza correspondiente a la extracción quirúrgica en bloque del tejido disgenésico renal y el uréter desembocando ectópicamente en la dilatación quistica de la vesicula seminal derecha.

ureteral se localizará ectópicamente, pero además dicha yema ureteral no contactará adecuadamente con el blastema nefrogénico produciéndose distintos grados de disgenesia o agenesia renal en aproximadamente el 66\% de los pacientes con uréter ectópico ${ }^{12}$. Sin embargo, el porcentaje de asociación de disgenesia (hipoplasia, displasia o aplasia) o agenesia renal ipsilateral es mucho mayor cuando la ectopia ureteral afecta al tracto seminal, con series que alcanzan el $90 \% 9$. De hecho, Das y cols. ${ }^{13}$ hablan de asociación invariable y clasifican la ectopia ureteral extravesical en dos tipos: ectopia del seno urogenital cuando el uréter desemboca en la uretra prostática y ectopia del conducto mesonéfrico cuando lo hace en alguno de sus derivados embriológicos que componen la vía seminal, sea la vesícula seminal, el conducto eyaculador, el conducto deferente o los 2/3 inferiores del epidídimo. Su disposición en la vesícula seminal ocurre en un 28-33\% del total de abocamientos ectópicos, lo que supone el $66 \%$ de las localizaciones en la vía seminal $^{2,14}$.

Por otra parte, un desarrollo anormal de la porción distal del conducto mesonéfrico puede causar la atresia congénita del conducto eyaculador $^{13}$, lo que daría lugar a una dilatación quística de la vesícula seminal tipo III de la clasificación de English de los quistes pelvianos ${ }^{15}$. La asociación de dilatación quística de la vesícula seminal con agenesia renal ipsilateral fue descrita por primera vez por Zinner en 1914. Lo que resulta aún más excepcional es la presencia añadida de un uréter ectópico desembocando en dicha vesícula seminal con dilatación quística como se describe en nuestro caso. Cherullo y cols. ${ }^{3}$ tras su revisión, aportaron el octavo caso descrito en la literatura.

Otras malformaciones que pueden estar asociadas serían reflujo vesicoureteral contralateral, riñón poliquístico, fístula traqueoesofágica $\mathrm{o}$ imperforación anal ${ }^{16}$. Así mismo se ha descrito la agenesia testicular o del deferente asociada a la vesícula seminal quística ${ }^{17}$. En la mayoría de los casos se trata de un varón de raza blanca en la $2^{\text {a }}$ ó $3^{\text {a }}$ década de la vida, la malformación es unilateral siendo más frecuente en el lado izquierdo y el uréter ectópico drena una vía urinaria única, con menos de un $8 \%$ de los casos afectando al pielón superior de una duplicidad ureteral ${ }^{2}$.

En cuanto a la clinica cuando hay ectopia ureteral, los sintomas son distintos según la localización del orificio ureteral, con diferencias significativas de acuerdo con la edad del paciente. Así, en varones menores de 15 años, hasta en un 74\% el uréter desemboca ectópicamente en el tracto urinario dando síntomas de infección y dolor lumbar. Sin embargo, por encima de los 15 años, en más del $65 \%$ el abocamiento del uréter ocurre en el tracto seminal produciendo síntomas de orquiepididimitis, dolor perineal o eyaculatorio ${ }^{9}$. Se explicaría como una irritación de los órganos adyacentes a la distensión quística de la vesícula por el acúmulo de secreciones seminales a partir de la pubertad. La palpación de una masa elástica paraprostática al 
tacto rectal es un hallazgo poco frecuente ${ }^{2}$ que resultó muy útil en la orientación diagnóstica de nuestro paciente en el que el único síntoma era un dolor inespecífico en la fosa iliaca derecha.

Entre las posibles patologias que precisan diagnóstico diferencial se han descrito el ureterocele, cáncer de recto, divertículo de colon, leiomioma vesical, quiste del conducto de Wolff o de Müller, quiste dermoide ${ }^{2}$, liposarcoma o absceso del fondo de saco de Douglas. Para ello la mejor prueba de imagen inicial es la ecografia abdominal y transrectal ${ }^{3}$, que permite orientar el diagnóstico diferencial de una masa quística pélvica según su relación con el cuello vesical de modo que si es lateral, puede tratarse de un quiste de la vesícula seminal ${ }^{18}$.

En la urografia intravenosa casi es constante la anulación funcional renal ipsilateral. La cistoscopia aporta la ausencia de orificio ureteral en el hemitrígono correspondiente ${ }^{2,3}$. La TC muestra anomalías a nivel renal o de la vesícula seminal pero puede resultar insuficiente para confirmar el diagnóstico ${ }^{14}$. Otras pruebas como la deferentovesiculografia o la punción del quiste tampoco suelen aclarar el nivel de inserción del uréter en la vía seminal $^{2,16}$, además del riesgo de complicaciones como la infección yatrogénica en nuestro paciente en el que no se detectó la presencia de espermatozoides en el aspirado quístico descrita en la literatura $^{13}$. Otra complicación sería la posible alteración de la fertilidad ${ }^{5}$ que en muchos casos está conservada gracias a la normalidad de la vía seminal contralateral. Nuestra conclusión de acuerdo con otros autores $^{5,6,14}$, es que la RM es la mejor prueba de imagen para obtener un claro diagnóstico prequirúrgico de forma no invasiva y que debe ser de elección ante la sospecha de esta compleja patología.

El hallazgo incidental en pacientes asintomáticos solo requiere un seguimiento, reservando la cirugía para los casos sintomáticos ${ }^{2}$. La vía laparoscópica ha sido recomendada por autores con experiencia aportando una baja morbilidad quirúrgica ${ }^{3}$. Sin embargo, la mayoría de grupos realizan una resección en bloque por abordaje retroperitoneal abierto del riñón displásico, el uréter y la vesícula seminal, que creemos aún más indicada para facilitar la cirugía si se han producido adherencias por cambios inflamatorios sobreañadidos como ocurrió en el caso que aportamos.

\section{REFERENCIAS}

1. Sheih CP, Hung CS, Wei CF, Lin CY. Cystic dilations within the pelvis in patients with ipsilateral renal agenesis or dysplasia. J Urol 1990;144:324-327.

2. Berne Manero JM, Bono Ariño A, Roncales Badal AL. Retroiliac ectopic ureter opening to the seminal vesicle. Arch Esp Urol 2001;54(1):61-64.

3. Cherullo EE, Meraney AM, Bernstein LH, Einstein DM, Thomas AJ, Gill IS. Laparoscopic management of congenital seminal vesicle cysts associated with ipsilateral renal agenesis. J Urol 2002;167(3):1263-1267.

4. Arey LB. Developmental Anatomy, $7^{\text {th }}$ ed. Philadelphia, WB Saunders 1966:324-340.

5. Callewaert P, De Coster M, Vuylsteke P, De Man R, Brijs S, Baert L. Anal tenesmus caused by seminal vesicle cyst. Urology 1997;49(1):139-141.

6. Roberts SG, García Mediero JM, Segura JW, Rivas JA, García Alonso J. Incidental pelvic mass identified during ultrasound-guided transrectal needle biopsy of the prostate. Arch Esp Urol 2002;55(4):466-468.

7. Schulman CC. L'association des malformations rénales et urétérales. Une hypothèse embryogénique. 70 Congrés de L'A.F.U. Procès verbaux, memories et discussions. Masson édit. Paris 1976:420-423.

8. Nakachi K, Kuroda J, Terakawa T, Miyamoto I, Takemura T. Ectopic ureteral opening in the male: two case reports. West Japan Urol 1989;51:149-152.

9. Terai A, Tsuji Y, Terachi T, Yoshida O. Ectopic ureter opening into the seminal vesicle in an infant: a case report and review of the japanese literature. Int J Urol 1995;2(2):128-131.

10. Bauer SB, Perlmutter AD, Retic AB. Anomalies of the upper urinary tract. Campbell's Urology, $6^{\text {th }}$ ed. Philadelphia, WB Saunders Co. 1992:1402-1442.

11. Röhrborn C, Schneider HJ, Alles JU, Engstfeld J. Embriologic and diagnostic aspects of associated malformation of upper urinary and genital tracts. Urology 1983; 22:588-593.

12. Gotoh T, Takahashi Y, Kumagai A et al. Renal dysplasia simulating epididymal structure: report of five cases and its embryogenesis. Clin Urol 1983;37:799-804.

13. Das S, Amar AD. Extravesical ureteral ectopia in male patients. J Urol 1981;125:842-846.

14. Matsuki M, Matsuo M, Kaji Y, Okada N. Ectopic ureter draining into seminal vesicle cyst: usefulness of MRI. Radiat Med 1998;16(4):309-311.

15. English V. Citado por HART JB.: A case of cyst or hydrops of the seminal vesicle. J Urol 1961;86:137.

16. Soler Fernández JM, Domínguez Bravo C, Herrera Puerto J, Murillo Mirat J, Castaño Casaseca JL, Poveda Pierola A. Uréter ectópico en vesícula seminal con displasia quística. Caso clínico y revisión de la literatura. Actas Urol Esp 1990;14(6):447-450.

17. Ornstein MH, Kershaw DR. Cysts of the seminal vesicle are Müllerian in origin. JR Soc Med 1985;78:1050-1051.

18. Dahms SE, Hohenfellner M, Linn JF, Eggersmann C, Haupt G, Thuroff JW. Retrovesical mass in men: pitfalls of differential diagnosis. J Urol 1999;161:1244-1248.

Dr. M. Pascual Samaniego

Clemente Sánchez Ramos, 1 - 5으 B

10001 Cáceres

mpaski10@yahoo.es

(Trabajo recibido el 15 diciembre de 2003) 